\title{
Heat Transfer Analysis of Plate-fin Heat Sink with Piezoelectric Fan
}

\author{
Yen-Tso Chang ${ }^{1, *}$, Han-Ching Lin ${ }^{1}$, Chi-Jui Huang ${ }^{1}$, Chun-Hsien Chen ${ }^{2}$, Chien-Jen Lin ${ }^{3}$, Go-Long Tsai ${ }^{1}$ \\ ${ }^{1}$ Graduate Institute of Mechanical and Electrical Engineering, National Taipei University of Technology, Taiwan \\ ${ }^{2}$ Graduate Institute of Education Entrepreneurship and Management, National University of Tainan, Taiwan \\ ${ }^{3}$ Department of Vehicle Engineering, National Taipei University of Technology, National University of Tainan, Taiwan
}

Copyright $@ 2015$ Horizon Research Publishing All rights reserved.

\begin{abstract}
Applying software Ansys-Fluent to analysis the effect of piezoelectric fan device installed inside rectangular channel by numerical simulation method. The piezoelectric fan is activated by reversible piezoelectric effect in piezoelectric material, change rectangular channel flow field construction, then it affects heat flow status in advance. Numerical simulation parameter are included Nusselt number $(\mathrm{Nu})$, distance between peak of fan blades to front part of heat $\operatorname{sink}\left(L_{g}=0,5,10,-11.25,-22.5,-33.75,-45\right)$, height from center on piezoelectric fan bottom side $\left(H_{w}=10\right.$, $16,21)$, number of piezoelectric fan (single-fan and twin-fan), phase-shift (in-phase and counter-phase), and numbers $(n=10,14)$ for heat sink fin. This result is indicated that good position to temperature dropped greatly is located from the front part of piezoelectric fan to the front of heat sink $\left(L_{g}=-22.5\right)$ under fixes distance $\left(H_{w}=21\right)$ from piezoelectric fan to channel also found to provide double piezoelectric fan heat drop effect is equal to single one, however, double piezoelectric fans would provide higher heat dissipation as Counter-phase. The performance of piezoelectric fan is improved depends on change height $\left(H_{w}\right)$ from center of piezoelectric fan to channel bottom; the height $\left(H_{w}\right)$ shall be decreased as piezoelectric fan placement at the front of heat sink.
\end{abstract}

Keywords CFD, Electronic Cooling, Piezoelectric Fan, Plate-fin Heat Sink

\section{Introduction}

With advances in semiconductor process technology and development, electronic components toward slim and light design concept, the challenge facing the problem of overheating the more gradual increase. At the same size, $\mathrm{CPU}$ (Central processing unit) the number of transistors inside the integrated circuit with the progress of the process and increase, which also represents the chip under the heat of the same size also increases; while the same size in integrated circuits doubles every eighteen months will enhance the efficiency; this also means the chip under the heat of the same size will also increase, will result in the operation of the electronic components, the heat generated per unit area are constantly rising. Because of integrated circuits get smaller, making a wide range of portable consumer electronics product innovation in mobile phones, laptop computers a common design philosophy is to enhance user comfort and low noise level, can be low power down time and extend the operating life of the product, research and development of cooling devices such products as design criteria, in response to the advent of high- power and high-density electronics equipment age, and to enable them to function properly in a safe operating temperature how to effectively enhance the thermal performance of electronic components equipment in recent years became a very important research topic.

For heat sink, Naik et al. [1] in the rectangular flow channel flat ribs to conduct heat transfer characteristics, the local heat transfer coefficient distribution along the height of the ribs for a limited range of geometries indicated that its magnitude and profile was highly influenced by the amount of clearance above the ribs. Lower heat transfer coefficients ensue when a clearance gap is introduced above the ribs, which varies from an almost constant value along most of the rib height to a maximum value at the rib. El-Sayed et al. [2] varied the fin-height, fin-width, space between fins, fins-number and the distance of fin tips from the shroud to investigate the performance of plate-fin heat sink; their conclusions were that the pressure drop increases with increasing Reynolds number and fin height but decreases with increasing inter-fin space and fin width, that increasing the clearance between the fin tips and the shroud decreases the mean Nusselt number, and that the mean Nusselt number increases with increasing Reynolds number, fin width and space between fins but with decreasing fin height. El-Sayed et al. [3] studied the heat transfer and fluid flow of a plate-fin heat sink for the cases of parallel flow, impinging flow and reverse impinging flow; a parallel flow yields the greatest rate of heat transfer and the least pressure drop. Tsai et al. [4] 
investigated the effect of the angle of inclination of a plate heat shield on the thermal and hydraulic performance of a plate-fin heat sink. The research discovered that the sloping baffle is unable to reduce the thermal resistance, but can actually reduce the pressure drop effectively. As a compromise between the demands of small thermal resistance and a small pressure drop, the angle of inclination of a plate heat shield must be chosen carefully.

Choi et al. [5] fix frequency of piezoelectric fan at $180 \mathrm{~Hz}$, use both testing method and 2-D numerical simulation method for research vorticity induced by piezoelectric fan swinging, in research speed intensity after 40 periods opens to be as mesh analysis for research of numerical simulation, it's to be individual once mesh number increases to 58,890 meshes. In research, the result indicates obviously that numerical-simulation analysis flow to discovery the area of flow field is the same compared to testing method, but vorticity is smaller than from testing method, numerical-simulation method can resolve vorticity characteristic data, also realized there have four-step to induce vorticity: initiation, development, separation and propagation. Buermann et al. [6] apply topology to analyze flow field from piezoelectric fan, as above mention, piezoelectric fan is equipment with advantage of low-power, tiny, and low noise...etc, that easy to apply on portable electric product. They analyze effect resulted from piezoelectric material by Bernoulli equation and Finite element, this analysis provide perfect length ratio of piezoelectric fan and thickness on blade top for max. EMCF (Electromechanical coupling factor), EMCF that means to get machine power under deformed cycle, piezoelectric material in quasi-stratic, both tip deflection and rotation are key parameter mainly impact performance, easy to design base on this optimization, piezoelectric fan promote design with combine and fan mode, the vorticity is induced by blade of fan around while moving blade horizontally swing, the leave fan from tip of blade. Later Acikalin et al. [7] the use of piezoelectric fan with a small size, low noise and low power consumption advantages of the use of the electronic component, but does not replace the current market piezoelectric fan axial rotation of the fan for the purpose, but in the shaft local area using a piezoelectric fan temperature under high flow fan cooling effect not play situations. Experimental results show that when the vertical piezoelectric fan placed in a central location on the phone module, the heat transfer coefficient and superior level better placement. Liu et al. [8] studied the vertical and horizontal piezoelectric fan placed on the flat surface of the heat transfer effects of heat, and experimental methods for the use of six different geometries piezoelectric fans, studies found that the heat transfer efficiency is increased because each both sides of the jet stream and pressure cycles generated by fan blade tip entrained airflow caused by the successive accumulation, while the vertical placement of the piezoelectric fan blades optimum heat transfer at the center position is symmetrical on both sides of the distribution. If the fan placed horizontally, the heat transfer performance degradation will be quite intense, because space is tight due to entrained air flow caused by partial blockage. Fans level of heat transfer performance is not placed below vertical display, which is also proportional to the distance from the surface of the plate. So Acikalin et al. [9] to flow visualization experiments way to assess the feasibility of the pressure applied to the electronic fan cooling system, using three sets of piezoelectric fan placed vertically and placed in a piezoelectric fan level to explore its transfer heat transfer coefficient, piezoelectric fan finally explore the feasibility of electronic components used in thermal performance. Abdullah et al. [10] put pressure on the level of form fan-dimensional analysis and numerical simulation of flow visualization experiments, observations explore the phenomenon of piezoelectric fan and their ilk field formed by the heat transfer coefficient. While high piezoelectric fan from the heat source to explore for; their optimum cooling performance can be reduced $70^{\circ} \mathrm{C}$, but the experimental and numerical analysis error of about $11 \%$. Wait et al. [11] using three different lengths to simulate the pressure of fans and flow visualization to three piezoelectric fan flow visualization model and validate each other and numerical simulation analysis to explore the resonance frequency of the piezoelectric fan arising under each modality and analyzed. The results showed that the fan under higher frequency resonance mode electromechanical coupling coefficient have better performance, but actually used in electronic cooling system for an extra considerations commercial specifications.

In this paper, we changed the flow structure to enhance heat transfer effect by utilizing the piezoelectric fan which is set in front of the radiator to mix the air surrounding radiator and the fins. Most of the current scholar used to change the fin shape to improve the performance of plate-fin heat sink, and vortex generator for the multi-fin-tube heat exchanger. As piezoelectric fan vibration at different position, affect different the flow construction, the main effect on heat sink is from jet flow and entrained flow induced by piezoelectric fan former and surface, that has significant differential as two phase vibration [12, 13]. Only few studies install piezoelectric fan for plate-type heat sink to enhance heat transfer effect. This study intended to develop a design with low thermal resistance, and to provide a new design direction for the present plate-fin heat sink cooling module design.

\section{Geometric Model}

\section{Calculation and Parameters}

Numerical simulation parameters rang is $205 \mathrm{~mm} \times 45 \mathrm{~mm}$ $\times 100 \mathrm{~mm}(\mathrm{X} \times \mathrm{Y} \times \mathrm{Z})$, geometric model illustrated in Figure1. This paper to uses single-fan and twin-fan both are investigated at positioned vertically in rectangular channel, analysis effects of plate-fin heat sink characteristic on piezoelectric fan with variable parameter value in the study. 
Red dot on fan shown in Figure 2, where is original point. Parameter values in the study as following: $\left(L_{g}\right)$ : Distance from fan tip (red dot) to heat sink, $\left(H_{w}\right)$ : Distance from center of fan to channel bottom, $(a)$ : Gap between Twin-fans is assumed $30 \mathrm{~mm},(n)$ : number of heat-sink fin and Vibration Phase: In-phase and Counter-phase.

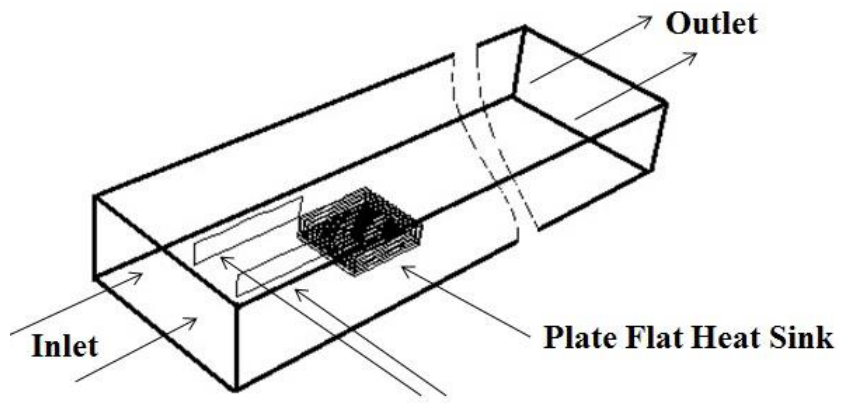

Piezoelectric Fan

Figure 1. Schematic diagram
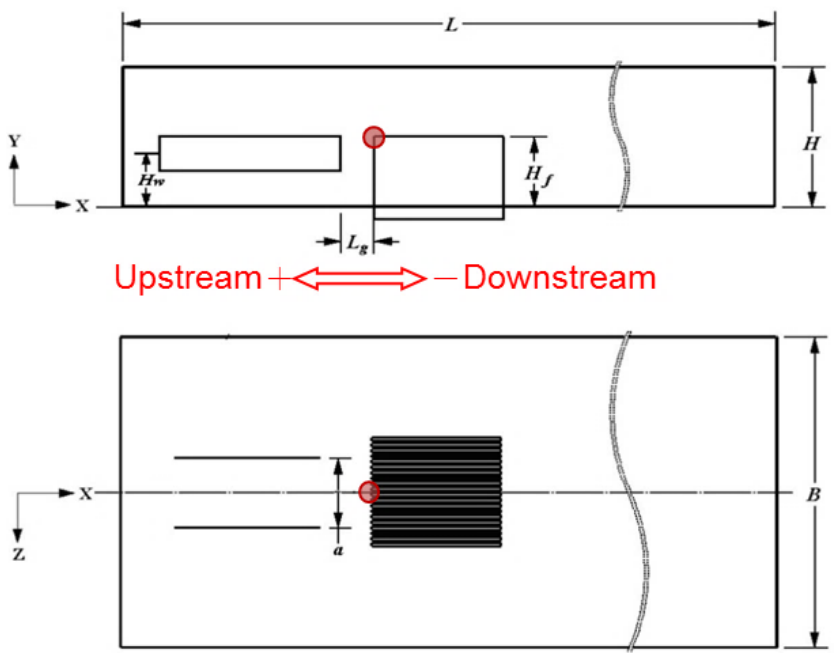

Figure 2. Parameters diagram

In order to simplify both question and analysis step, thus, assumes system as steady-state, fluid characteristic is incompressibility, neglect gravity and effects of thermal radiation. The boundary conditions are provided as: (inlet) $U=U_{0} \quad, \quad V=W=0 \quad, \quad T=T_{0}=293 K \quad, \quad$ (well) $U=V=W=\frac{\partial T}{\partial n}=0$, (outlet) $\frac{\partial \phi}{\partial n}=0, \phi=(U, V, W, \kappa, \varepsilon, T)$, Heat surface and solid heat transfer boundary conditions. Bottom of heat sink are thermally isolated except heater surface $(31 \mathrm{~mm} \times 31 \mathrm{~mm})$, heater surface heat to $35 \mathrm{~W}$, heat transfer coefficient of heat sink is $168 \mathrm{~W} / \mathrm{mK}$. Heat sink and fluid flow boundary conditions: parts non-isolated on heat sink, temperature of cross section on solid and fluid and thermal heat flux both are continuous. Piezoelectric fan boundary condition is isolation.

Single-fan produces approximately $2.5 \mathrm{~m} / \mathrm{s} \max$. Flow velocity by fan tip, overall dimensions of single-fan is 65 $\mathrm{mm}$ length, $12 \mathrm{~mm}$ width and vibration amplitudes is 25.4 $\mathrm{mm}$. For simplify analytical models, neglect thickness of piezoelectric fan during simulating. Vibration frequency is
62.5 Hz, fan vibration amplitudes refer to following equation:

$$
\begin{aligned}
& f(x)=-42.3402 x^{2}+33587.5 x^{3}-2.7317 \times 10^{6} x^{4}+ \\
& +9.05342 \times 10^{7} x^{5}-1.2653 \times 10^{9} x^{6}+6.34496 \times 10^{9} x^{7}
\end{aligned}
$$

$f(x)$ is for fan vibration amplitudes, $x$ is for independent variables, derive relation between time constant and $62.5 \mathrm{~Hz}$ vibration amplitudes from

$$
f(t)=f(x) \times \sin (2 \pi \times f r \times t)
$$

Where $f(x)$ is for fan vibration amplitudes, $f r$ is for frequency, $t$ is time, these three parameters are applied by "FLUENT" software compiled with user-defined function (UDF). There are two behaviors of Twin-fan oscillation, Counter-phase and In-phase, please see Figure 3

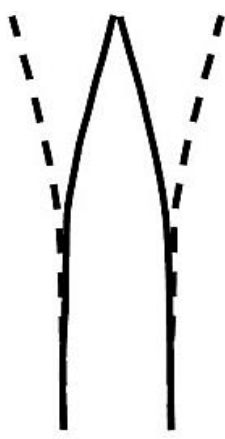

Counter-phase

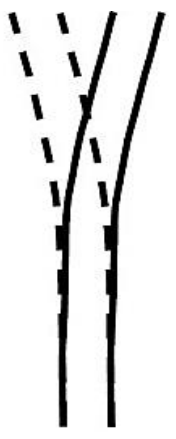

In-phase
Figure 3. Counter-phase and In-phase parameters diagram

\section{Numerical Method}

Previous processor GAMBIT establishes model mesh and defined as model boundary condition, then transfer mesh file to FLUENT software. Initially FLUENT import mesh file to makes grid check, and makes smooth/Swap mesh, Hybrid meshing method is used in this study, which is equal to mesh constructed by tetrahedron mesh and hexahedral mesh. Heat sink and rectangular channel constructed as hexahedral mesh, and piezoelectric fan is constructed as tetrahedron mesh; then determines solver and laminar flow model, and set boundary condition parameter, inlet pressure, heating flux and material characteristic.

As applied the first law of thermodynamics, you should define the space contained in a controlled surface area and allow this energy and matter could enter and leave the surface, this area was called the controlled volume. Using the heat transfer rate equation to represent the conservation of energy, the formula could be written as

$$
\dot{E}_{\text {in }}+\dot{E}_{g}-\dot{E}_{\text {out }}=\dot{E}_{s t}
$$

Generated heat $\dot{E}_{g}$ was in the controlled volume, the energy conversion heat generated during this study. There was no energy conversion, and therefore this was ignored. $\dot{E}_{\text {in }}$ and $\dot{E}_{\text {out }}$ represented heat and mechanical energy 
reacted in and out of the controlled volume, storage term $\dot{E}_{s t}$ meant storing energy within the physical changed. From (2) the energy per unit volume of the time rate of change could be rewritten as,

$$
\iiint_{C . V} \rho c_{p} \frac{\partial T}{\partial t} d x d y d z+h_{c} A_{t}\left(T_{a v e}-T_{\infty}\right)-\dot{E}_{i n}=0
$$

where $\rho$ is density and $C_{p}$ was specific heat, the product was known as volumetric heat capacity, $\frac{\partial T}{\partial t}$ was the energy per unit volume within the object of the time rate of change, $d x d y d z$ represented the control volume, At was the total cooling area of heat sink, $h$ was the average heat transfer coefficient, $\left(T_{\text {ave }}-T_{\infty}\right)$ was for the temperature difference of controlled surface and volume of the fluid.

$C o p_{v}$ expressed the coefficient of performance, this amount represented the dimensionless unit of time which took away the heat and power consumption, copv could be written as $\operatorname{Cop}_{v}=R_{t h, n d} / R_{t h, p z f}$, where $R_{t h, p z f}$ was the piezoelectric fan thermal resistance, $R_{t h, n c}$ was the case of natural convection heat sink thermal impedance. This impedance ratio obtained from piezoelectric fan which reflected indicators of the overall thermal performance.

In study, for the pressure parameter is adopted by Standard method, and for pressure associates to velocity which is adopted by reliable method SIMPLE-C to appropriate for laminar flow model [14], using pressure equation that is derived from continuous equation to correct pressure drop. Increasing Relaxation factor in calculation, then dynamic and energy equation can be used to first order upwind scheme in order to initialize velocity and pressure at $X 、 Y$ 、 $\mathrm{Z}$ direction respectively, then determine convergence criterion of velocity, energy equation and continuous equation, define as average temperature in entrance. In this work, a numerical analysis model established using the computational fluid dynamic software, to simulate the piezoelectric fan behavior of vibration. A convergence criterion in this numerical analysis is defined as energy equation of Scaled Residual which is less than $10^{-8}$, and Scaled Residual for others equation is less than $10^{-5}$.

\section{Result and Discussion}

In this study, we use a numerical analysis to investigate effect of thermal and flow fields on plated heat sink and piezoelectric fan installed in rectangular channel. Set up heating flux $35 \mathrm{~W}$ to heat bottom of heater to unsteady simulate temperature raise for CUP of desktop computer, activating piezoelectric fan as temperature raising after 40 seconds, cooling time is 2 seconds. To investigate thermal flow efficiency of heat sink under conditions with different parameter.

For analysis, neglect temperature gradient in plate-fin heat sink, defines distribution of unsteady temperature per energy balance of objective. Discussion with simulating for following parameters: $\left(L_{g}\right),\left(H_{w}\right),(a)$, (single-fan and twin-fan), (counter-phase and in-phase) and (n).

\section{Effect of Single Piezoelectric Fan}

Distance between different piezoelectric fan to tip of plated heat sink relatively compared with slope of temperature shown in Figure 4, as position of piezoelectric fan located $L_{g}=-22.5 \mathrm{~mm}$, gets lower temperature slope. Effect of heat transfer on heat sink is decreased when position of piezoelectric fan varies with $L_{g}$, result in temperature slope increased. Piezoelectric fan is in front of heat sink, $L_{g}=10 \mathrm{~mm}$, temperature slope has the most significant influence. Impact flow is induced by piezoelectric fan lead to dynamics are not able to be transferred to heat sink, bring about temperature slope suddenly increasing. As $L_{g}$ is less than $10 \mathrm{~mm}$, piezoelectric fan position does not significantly affected heat transfer on heat sink; As piezoelectric fan is activated at 0.5 second, temperature slope is to be stable gradually, but curve is oscillation resulted from piezoelectric fan operates at $62.5 \mathrm{~Hz}$.

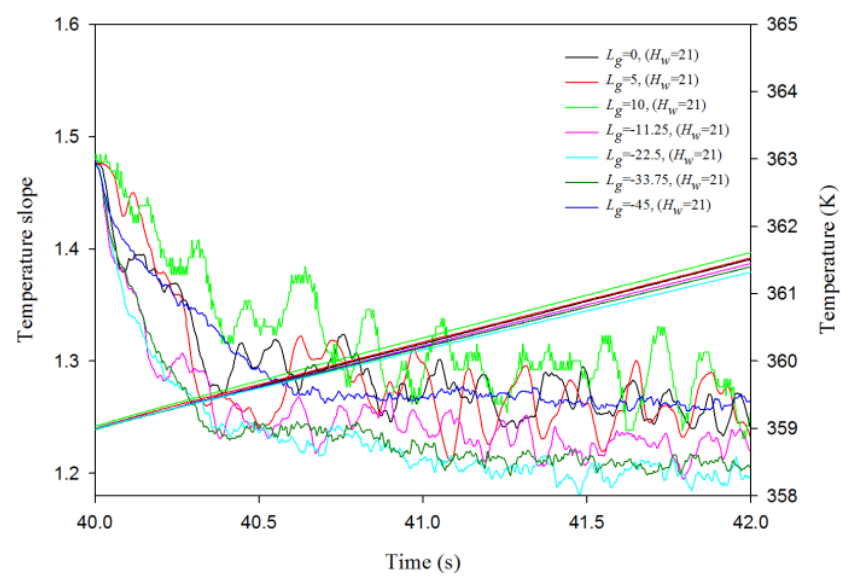

Figure 4. Temperature slop and temperature distributions for $L_{g}$. $\left(H_{w}=21\right.$ $\mathrm{mm}, n=14$, single-fan)

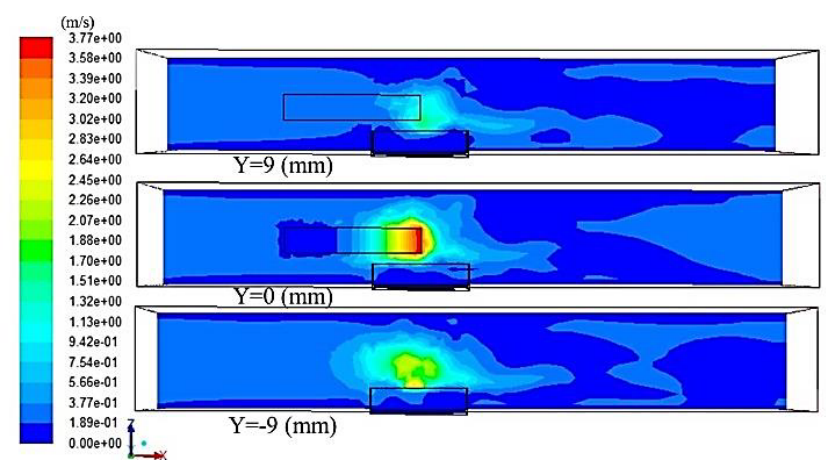

Figure 5. Contours of velocity magnitude in various cross-section along the Y-axis. ( $L_{g}=-22.5 \mathrm{~mm}, H_{w}=21 \mathrm{~mm}, n=14$, single-fan $)$

$L_{g}=-22.5 \mathrm{~mm}$ show in Figure 5, piezoelectric fan induces entrained flow that significantly affect heat sink, Impact flow in back of heat sink has formed to velocity boundary layer without much barrier, it occurs large convection effect on top of heat sink. Figure 6 presents lowest thermal resistance 
occurs at $L_{g}=5 \mathrm{~mm}$ with $H_{w}=10 \mathrm{~mm}$. The distance $\left(H_{w}=10\right.$ $\mathrm{mm}$ ) from piezoelectric fan center to bottom of flow channel, impact flow get heat not only transferred more efficiency and decrease thermal resistance but also improve thermal performance on heat sink. In other word, Channel inside channel of heat sink affect gradient to adversely influence heat transferring.

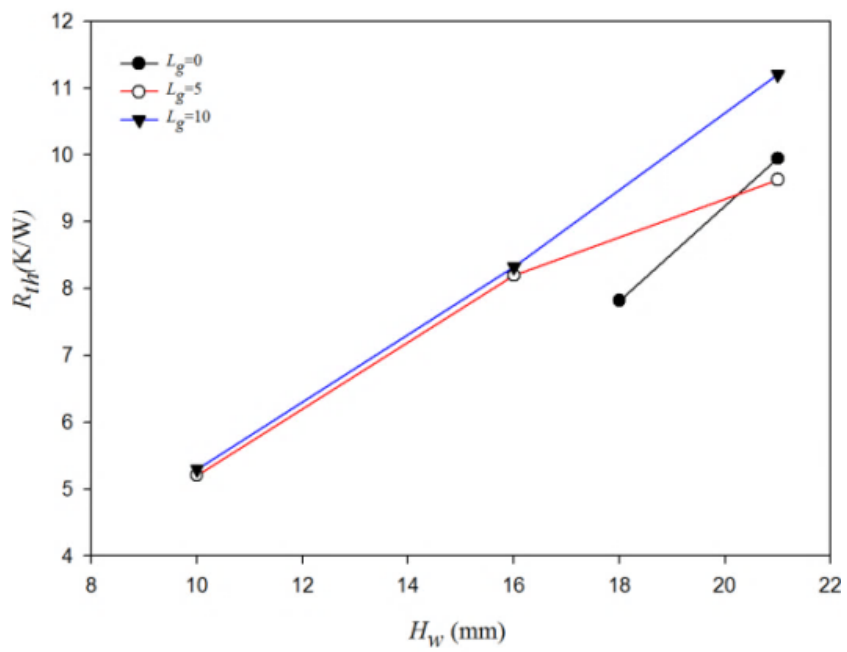

Figure 6. Variation of thermal resistance with $H_{w}$. $\left(L_{g}=0,5,10 \mathrm{~mm}, n=\right.$ $14, \mathrm{t}=42 \mathrm{~s}$, single-fan)

\section{Fin number}

Using coefficient of performance $C O P_{v}=\frac{R_{t h, n c}}{R_{t h, p z f}}, R_{t h, p z f}$ is thermal resistance provided by piezoelectric fan and $R_{t h, n c}$ is thermal resistance of the heat sink under natural convection, $C O P_{v}$ as $(n)=10$ and 14 , distance from fan center to bottom $\left(H_{w}\right)$ of flow channel is $21 \mathrm{~mm}$, investigate effect with variable distance $\left(L_{g}\right)$ from fan tip to in front of heat sink. See Figure 7, as $\left(H_{w}\right)=21 \mathrm{~mm}$, given distance $L_{g}=10,-22.5$, $-33.75,-45$; The result shown that coefficient of performance at $n=10$ is higher than at $n=14$; as given distance $L_{g}=-45$, the coefficient of performance at $n=10$ is higher than at $n=$ 14 as well.

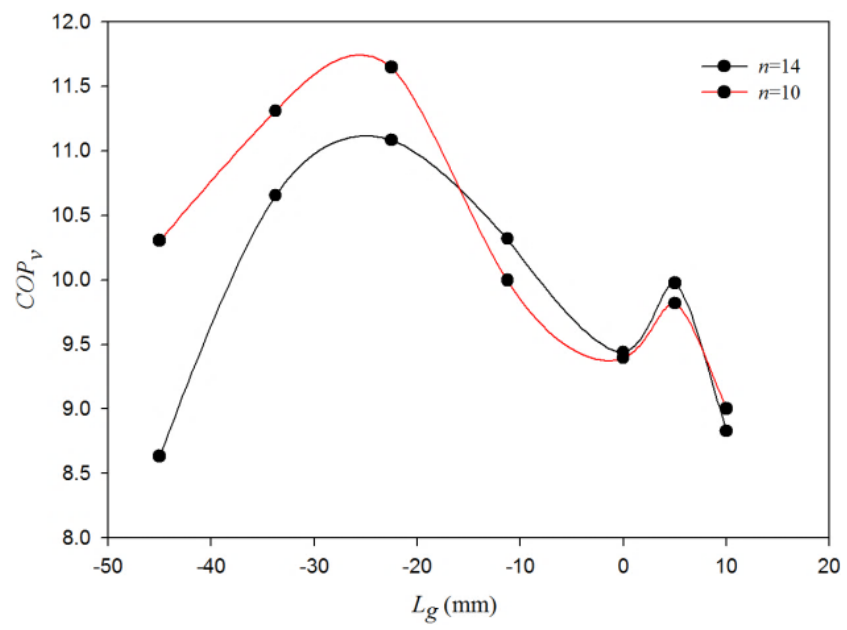

Figure 7. The effects of the $C O P_{v}$ on $L_{g}$. $\left(H_{w}=21 \mathrm{~mm}, t=42 \mathrm{~s}\right.$, single-fan $)$

Two parameter of fin number $n=14$ and $n=10$ have same $C O P_{v}$ at $L_{g}=10$, that is distance from piezoelectric fan tip to heat sink former fin, the position at $n=10$ provides $C O P_{v}$ greater than it at $n=14$. In fin number $n=10$ condition, piezoelectric fan tip produces energy can be transferred to heat sink inside, enhancing thermal fluid in heat sink. Take integral partial of thermal flux on surface of heat sink and defines as average is greater than fin number $n=14$. In this condition, twin-fan vibration by counter-phase, pressure differential occurs on piezoelectric fan. Take data of piezoelectric fan activated after 2 seconds, is also 125 period of vibration. Negative pressure exists among twin-fan which makes increasing velocity at $\mathrm{Y}$-axis, temperature on heat sink is transferred to direction of $\mathrm{Y}$-axis. It provides highest $C O P_{v}$ at $H_{w}=21 \mathrm{~mm}$, because that heat sink locates near piezoelectric fan to exist negative pressure.

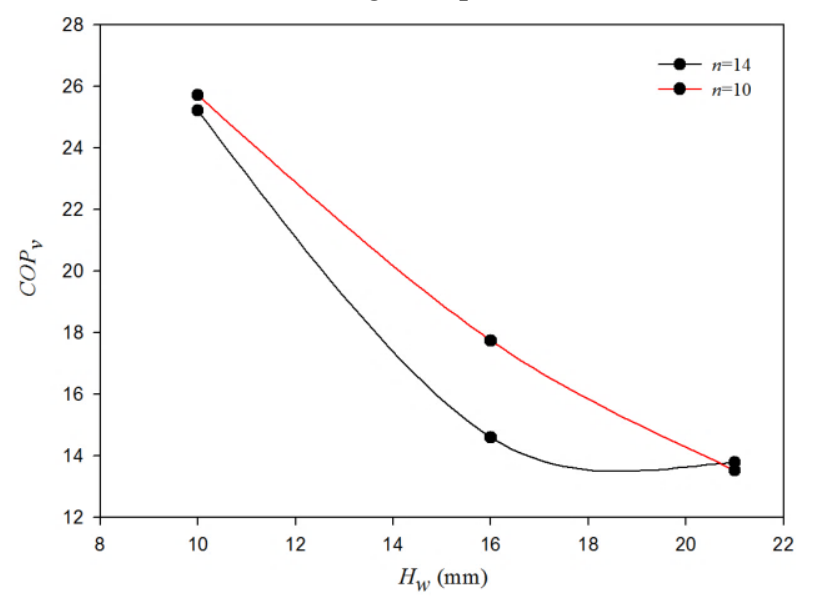

Figure 8. The effects of the $C O P_{v}$ on $H_{w}$. $\left(L_{g}=5 \mathrm{~mm}, t=42 \mathrm{~s}\right.$, twin-fan, counter-phase)

Figure 8 presents comparison plot of $C O P_{v}$ between variable piezoelectric fan to bottom of flow channel at $L_{g}=5$. As value $H_{w}$ is getting smaller, the $C O P_{v}$ is to be higher, there are not significant influence of increasing both $C O P_{v}$ and $(n)$ at $H_{w}=10$. Figure 9-10 present temperature distribution of $H_{w}=10$, at $n=10$ and $n=14$ respectively, streamline and vector on plot show heat transfer of heat sink inside, fluid makes temperature on heat sink inside is transferred to rectangular flow channel, impact flow directly impact former of heat sink, makes velocity of former of heat sink distributed toward Y-Z obviously. However, Twin-fan has with great performance of heat transfer on heat sink, but cost issue may be considered as use in electronic cooling system.

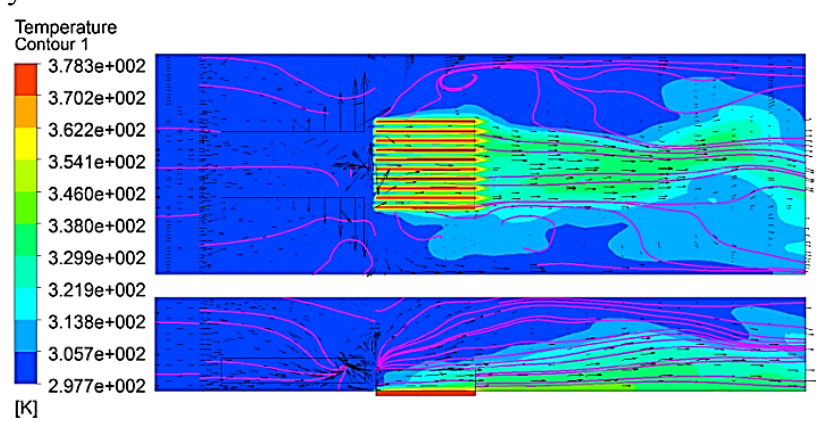

Figure 9. Contours of streamline and temperature, vector of velocity magnitude in $\mathrm{Y}=0$ and $\mathrm{Z}=0$ cross-section. $\left(L_{g}=5 \mathrm{~mm}, H_{w}=10 \mathrm{~mm}, n=10, t\right.$ $=42 \mathrm{~s}$, twin-fan, counter-phase) 


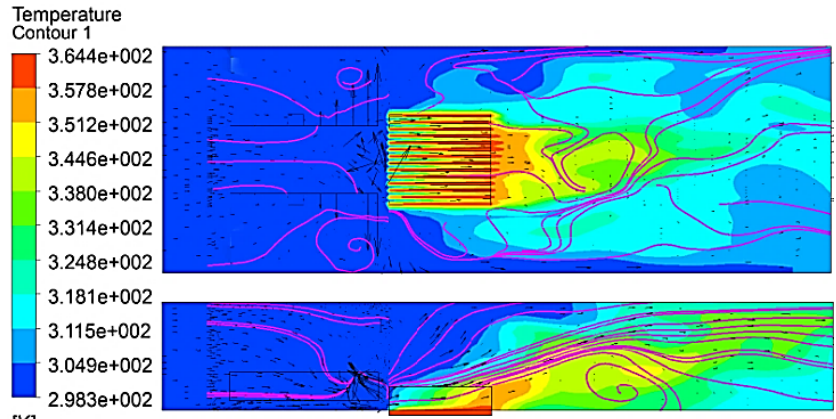

[K]

Figure 10. Contours of streamline and temperature, vector of velocity magnitude in $\mathrm{Y}=0$ and $\mathrm{Z}=0$ cross-section. $\left(L_{g}=5 \mathrm{~mm}, H_{w}=10 \mathrm{~mm}, n=14, t\right.$ $=42 \mathrm{~s}$, twin-fan, counter-phase)

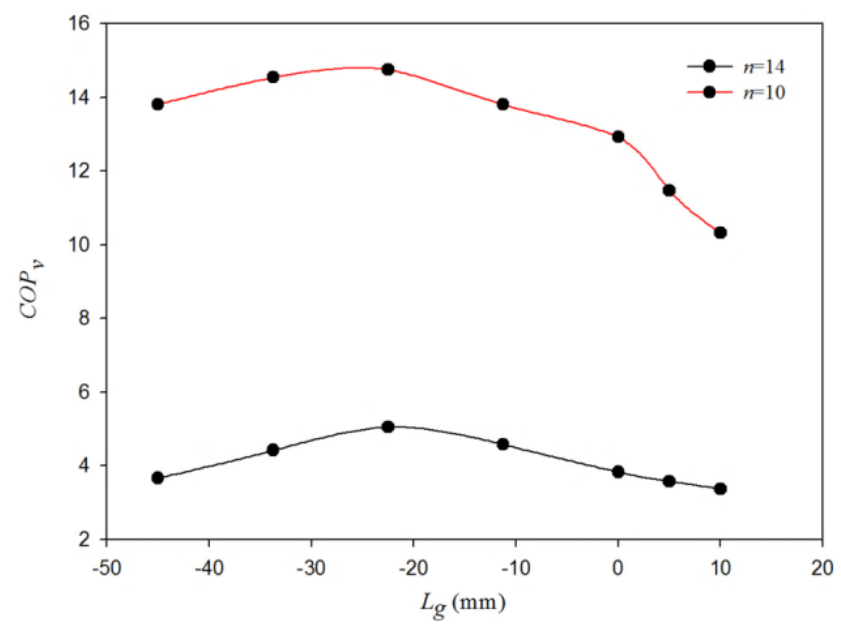

Figure 11. The effects of the $C O P_{v}$ on $L_{g} .\left(H_{w}=21 \mathrm{~mm}, t=42 \mathrm{~s}\right.$, twin-fan, in-phase)

Figure 11 presents comparison of $C O P_{v}$ of twin-fan vibration at in-phase at condition as to change $L_{g}$ at $H_{w}=21$. Maximum $C O P_{v}$ is given at this condition, it is higher at $n=$ 10 than at $n=14$ with different $L_{g}$. Figure 12 presents comparison of $C O P_{v}$ with change $H_{w}=21$ at $L_{g}=5$, piezoelectric fan is in-phase vibration, $C O P_{v}$ at $n=10$ higher than at $n=14$ with different $H_{w}$.

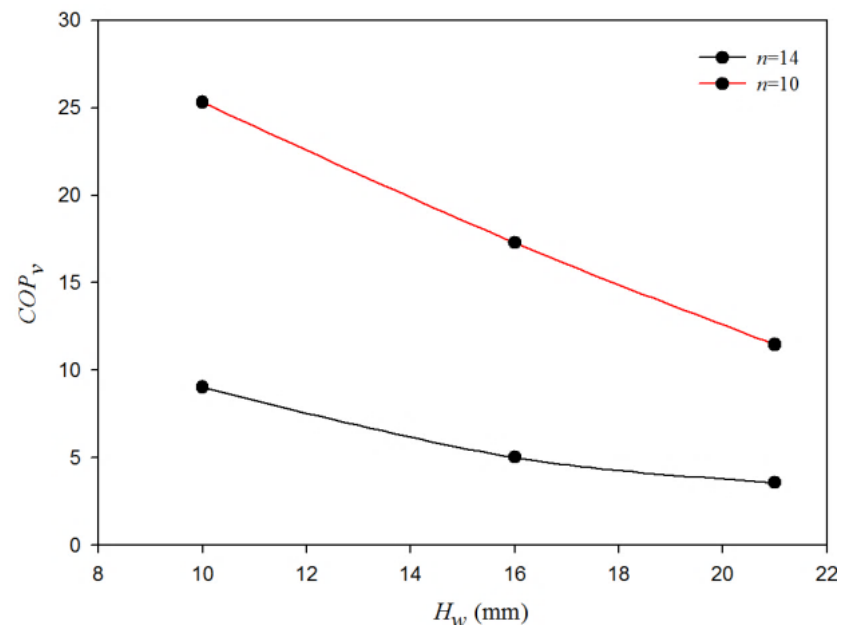

Figure 12. The effects of the $C O P_{v}$ on $H_{w}$. $\left(L_{g}=5 \mathrm{~mm}, t=42 \mathrm{~s}\right.$, twin-fan, in-phase)
Figs. 13-14 present temperature distribution at $n=10$ and $n=14$ at $L_{g}=-22.5$ respectively. Accumulating heat is apparent in former of heat sink area at $n=14$, velocity direct of back of heat sink tends to bottom of flow channel, the behavior advantage to spread heat to flow objective at lower temperature. However, Figure 15 presents distribution of temperature plot, streamline and vector both are shown concurrently that impact flow induced by twin-fan enters into channel of heat sink inside, and come to back of heat sink, make temperature transfer to pressure entrance. Figure 16 presents temperature distribution at same location with $n$ $=14$, fin-to-fin gap at $n=14$ is smaller than at $n=10$, flow resistance of heat sink is bigger at $n=14$, result in drop coefficient of performance since accumulating heat in back of heat sink.

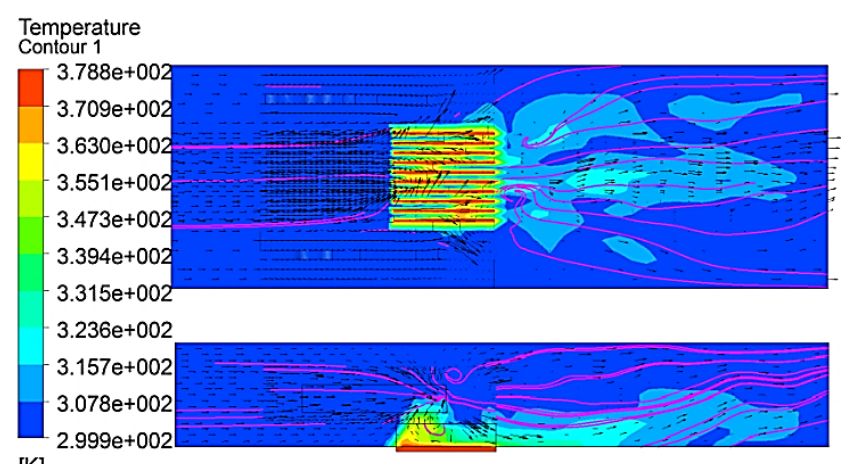

Figure 13. Contours of streamline and temperature, vector of velocity magnitude in $\mathrm{Y}=0$ and $\mathrm{Z}=0$ cross-section. $\left(L_{g}=-22.5 \mathrm{~mm}, H_{w}=21 \mathrm{~mm}, n=\right.$ $10, t=42 \mathrm{~s}$, twin-fan, in-phase)

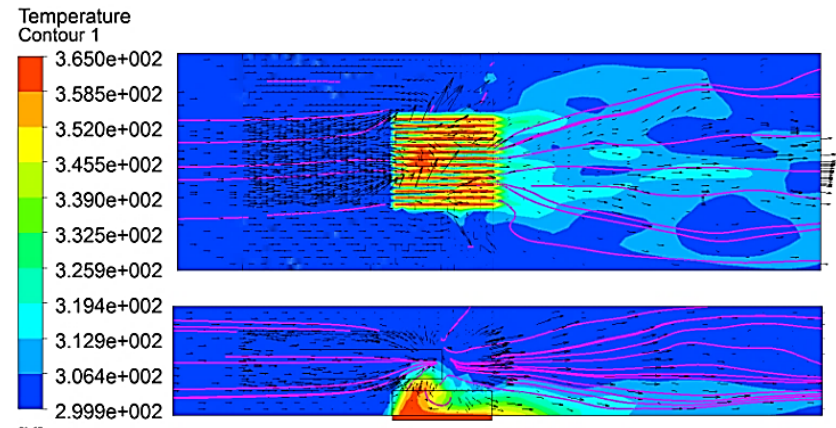

[K]

Figure 14. Contours of streamline and temperature, vector of velocity magnitude in $\mathrm{Y}=0$ and $\mathrm{Z}=0$ cross-section. $\left(L_{g}=-22.5 \mathrm{~mm}, H_{w}=21 \mathrm{~mm}, n=\right.$ $14, t=42 \mathrm{~s}$, twin-fan, in-phase)

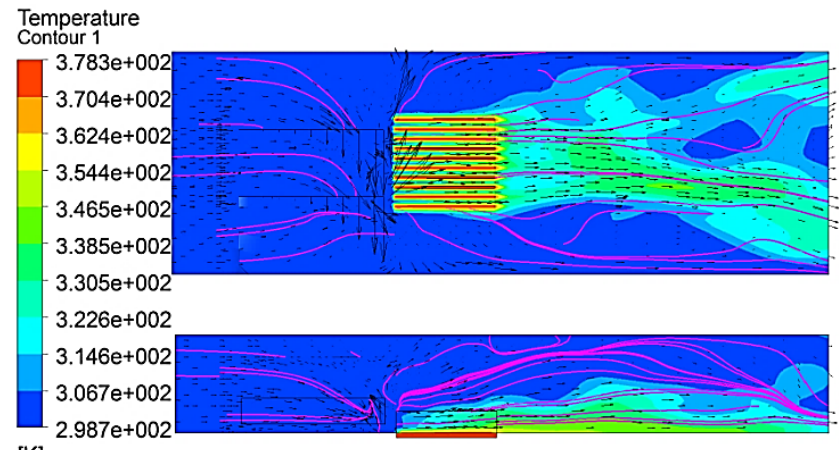
[K]

Figure 15. Contours of streamline and temperature, vector of velocity magnitude in $\mathrm{Y}=0$ and $\mathrm{Z}=0$ cross-section. $\left(L_{g}=5 \mathrm{~mm}, H_{w}=10 \mathrm{~mm}, n=10, t\right.$ $=42 \mathrm{~s}$, twin-fan, in-phase) 


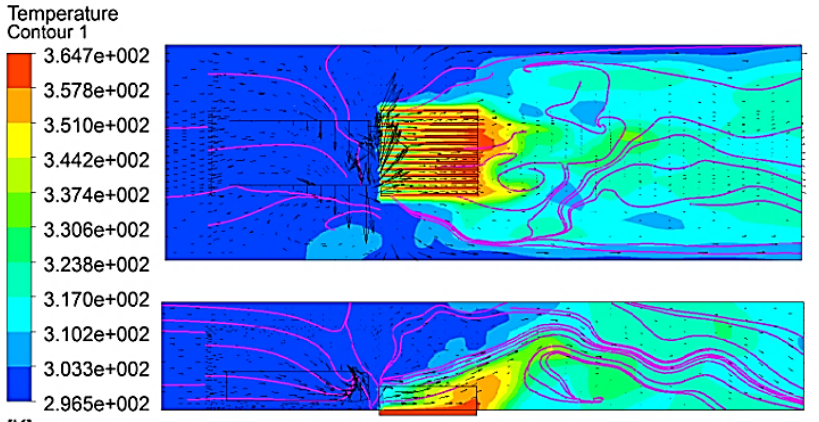

[K]

Figure 16. Contours of streamline and temperature, vector of velocity magnitude in $\mathrm{Y}=0$ and $\mathrm{Z}=0$ cross-section. $\left(L_{g}=5 \mathrm{~mm}, H_{w}=10 \mathrm{~mm}, n=14, t\right.$ $=42 \mathrm{~s}$, twin-fan, in-phase)

\section{Compare with In-phase and Counter-phase}

Heat transfer efficiency of counter-phase twin-fan is greater than single-fan, flow velocity at fan tip of either twin-fan or single-fan are the same, but volume flow rate of twin-fan is greater than single fan. In this study, investigate compared counter-phase with in-phase of $N u$, that shows convective flow occurred on surface, so get the relative convective flow with part of same fin number $n$ in twin-fan vibration.

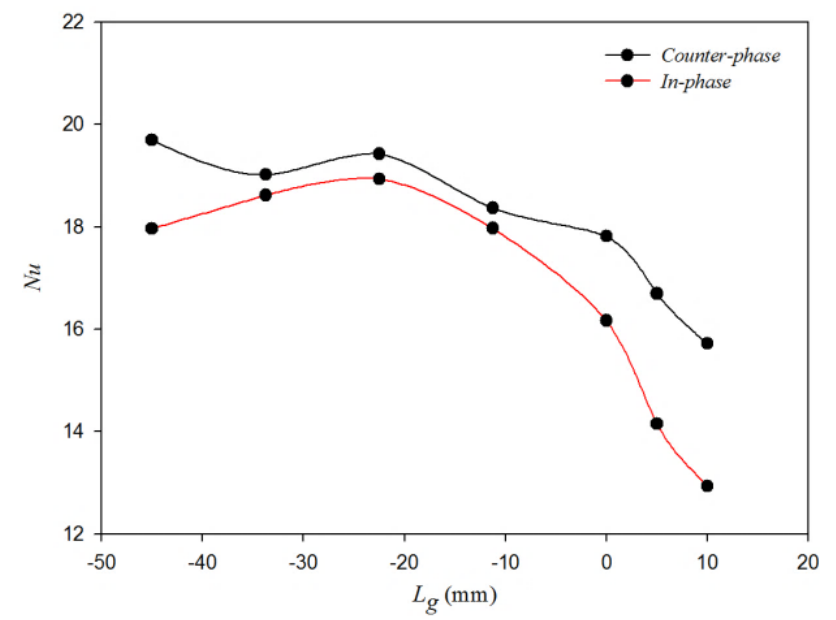

Figure 17. The effects of the Nusselt number on $L_{g}$. $\left(H_{w}=21 \mathrm{~mm}\right.$, twin-fan, $t=42 \mathrm{~s}, n=10$ )

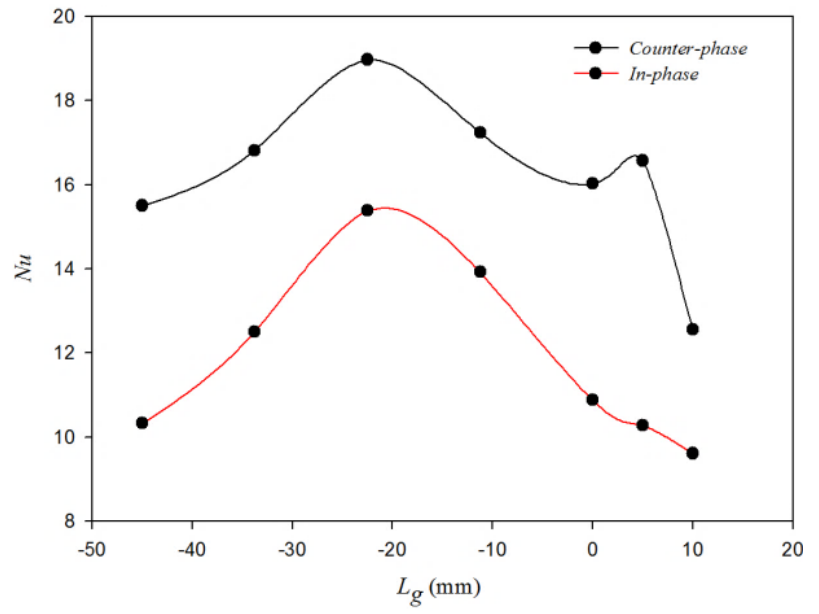

Figure 18. The effects of the Nusselt number on $L_{g}$. $\left(H_{w}=21 \mathrm{~mm}\right.$, twin-fan, $t=42 \mathrm{~s}, n=14$ )
Figure 17 presents $N u$ comparison of different distance of $\left(L_{g}\right)$ when fan vibration at counter-phase and in-phase. The convective flow performance at counter-phase is greater than in-phase on heat sink surface. When $L_{g}=0$, piezoelectric fan away from heat sink, convective flow is drop obviously at in-phase vibration, not much affect relative to counter-phase vibration. In condition of $n=14$, change parameter which distance from piezoelectric fan former to heat sink former $\left(L_{g}\right)$, heat sink surface of convective flow at twin-fan counter-phase vibration are greater than in-phase, best way of vibration at $n=10, L_{g}=-22.5$, see Figure 18 for details. This position, induced flow on twin-fan surface affect to advantage of heat transfer greater than jet flow.

When twin-phase is at counter-phase and phase angle 270 degree, negative pressure area locates between twin-fan that greater than vibration at in-phase. As twin-fan is at counter-phase, at $L_{g}=0$, fan tip impact flow affect work flow enters into fin inside and increase flow resistance, bring about heat transfer be lower than $L_{g}=5$, heat sink surface convective flow transfer getting decrease with piezoelectric fan moves away from heat sink. At $L_{g}=5$, change distance $\left(H_{w}\right)$ between center of piezoelectric fan to bottom of flow channel, twin-fan is at counter-phase makes $N u$ greater than in-phase. Figure out $N u$ number at fin number $n=10$ and $n=$ 14 in Figs. 19-20.

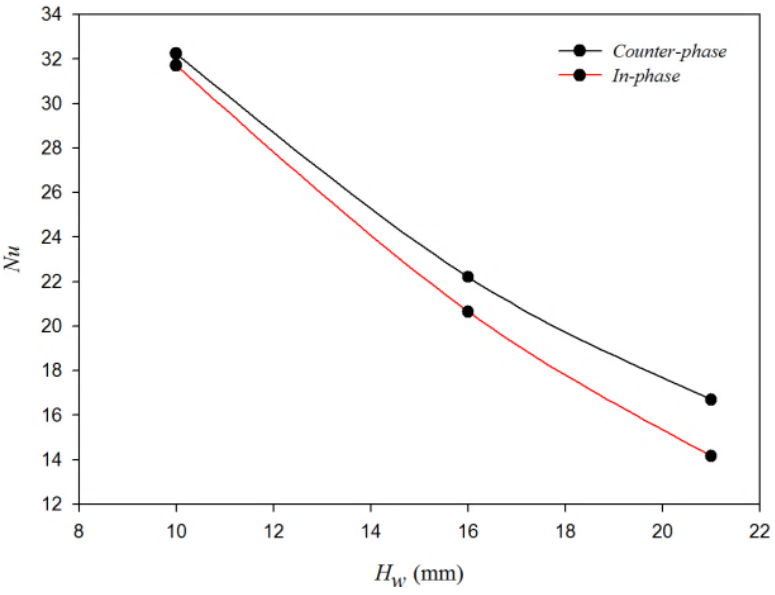

Figure 19. The effects of the Nusselt number on $H w$. $(L g=5 \mathrm{~mm}$, twin-fan, $t=42 \mathrm{~s}, n=10$ )

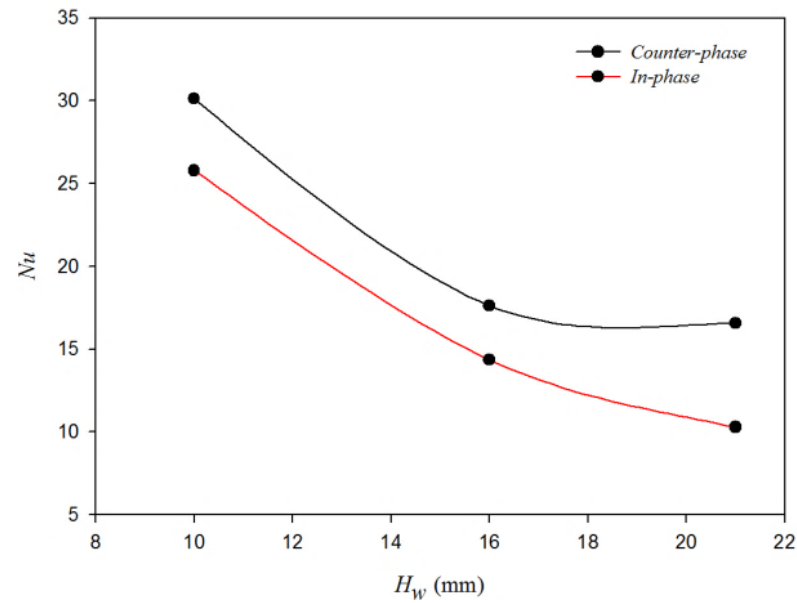

Figure 20. The effects of the Nusselt number on $H_{w}$. $\left(L_{g}=5 \mathrm{~mm}\right.$, twin-fan, $t=42 \mathrm{~s}, n=14$ ) 
Piezoelectric fan placed vertically, and the piezoelectric fan was placed in front of flat fins. As the piezoelectric fan was placed in different positions, right behind the heat sink fluid flow structure was somewhat different, but the impact of piezoelectric fan for the heat sink, that was mainly generated by the front end of jet flow and the surface of the entrained flow of piezoelectric fan, the two different placement of piezoelectric fan induced the disturbance moving gas for local cooling heat sink was obviously different. When the center position of piezoelectric fan was higher than heat sink fins, the affected area of entrained flow should be taken as a priority consideration. When the center position of piezoelectric fan was lower than heat sink fins, the effect of impact flow area should be taken as a consideration. The cooling performance increased as the fan centers to the flow channel height $\left(H_{w}\right)$ relatively decrease. At the occasion of piezoelectric fan placed at the front of heat sink, the closer distance between the centers of heat sink to the base plate of flow field, the better cooling performance it was. The piezoelectric fan would decrease it's cooling performance while fan base center to the flow channel height $\left(H_{w}\right)$ lower increased; when piezoelectric fan placed in front of the heat sink, the fan should make the fan center to the flow channel height lower, and the distance between the piezoelectric fan front to the heat sink fan front the closer the better, But at close range, should be avoided the bypass effect which was generated by the front end of the heat sink.

\section{Conclusions}

According to our numerical investigation of the effect of an inclined piezoelectric fan on the thermal and hydraulic performance of a plate-fin heat sink undergoing cross flow, we draw the following conclusions.

1. When piezoelectric fan center higher than fins, entrained flow area is priority of consideration, in study the most effect of entrained flow when location is at $L_{g}$ $=-0.5$ and $H_{w}=2.1$.

2. When piezoelectric fan center lower than fins, impact flow area is priority of consideration, in study the most effect of entrained flow when location is at $L_{g}=0.1$ and $H_{w}=1$. The performance of heat transfer varies with height $\left(H_{w}\right)$ that increase with height decreasing; It should makes decreasing height $H_{w}$ once piezoelectric fan position at in front of heat sink, the distance from piezoelectric fan tip to heat sink is as closer as better, but avoid bypass effect from impact flow induced by piezoelectric fan former within specify range.

3. One of advantage of the twin-fan in-phase is activated to make good performance of convective flow but only restricted around of fan, the flow efficiency is gradually decreasing with increasing distance from heat sink to fan, the other is that fan vibration at counter-phase is better heat transfer than vibration at in-phase.

\section{REFERENCES}

[1] S. Naik, S. D. Probert and I. G. Bryden. "Heat Transfer Characteristics of Shrouded Longitudinal Ribs in Turbulent Forced Convection," International Journal of Heat and Fluid Flow, Vol. 20 (4), pp 374-384, 1999.

[2] S. A. El-Sayed, S. M. Mohamed, A. M. Abdel-latif and A. E. "Abouda. Investigation of Turbulent Heat Transfer and Fluid Flow in Longitudinal Rectangular-Fin Arrays of Different Geometries and Shrouded Fin Array," Experimental Thermal and Fluid Science, Vol. 26 (8),pp 879-900, 2002.

[3] S. A. El-Sayed, S. M. Mohamed, A. M. Abdel-latif and A. E. Abouda. "Experimental Study of Heat Transfer and Fluid Flow in Longitudinal Rectangular-Fin Array Located in Different Orientations in Fluid Flow," Experimental Thermal and Fluid Science. Vol. 29 (8), pp 113-128, 2004.

[4] G. L. Tsai, H. Y. Li , and C. C. Lin. "Effect of the Angle of Inclination of a Plate Shield on the Thermal and Hydraulic Performance of a Plate-Fin Heat Sink," International Communications in Heat and Mass Transfer, Vol. 37, pp 364-371, 2010.

[5] M. Choi, C. Cierpka, and Y. H. Kim, "Vortex formation by a vibrating cantilever," Journal of Fluids and Structures, 2012, pp. 67-78.

[6] P. Bürmann, A. Raman, and S. V. Garimella, "Dynamics and Topology Optimization of Piezoelectric Fans," IEEE Transactions on Components and Packaging Technologies, 2003, pp. 592-600.

[7] T. Acikalin, S. V. Garimella, S. M. Wait, and A. Raman, "Exprimental Investigation of the Thermal Performance of Piezoelectric," Heat Tranfer Engineering, 2004, pp.4-14.

[8] S. F. Liu, R. T. Huang, W. J. Sheu, C. C. Wang, "Heat transfer by a piezoelectric fan on a flat surface subject to the influence of horizontal/vertical arrangement," International Journal of Heat and Mass Transfer, 2009, pp. 2565-2570.

[9] T. Acikalin, A. Raman, and S. V. Garimella, "Two-dimensional streaming flows induced by resonating thin beams," Acoustical Society of America, 2003, pp. 1785-1795.

[10] M. K. Abdullah, M. Z. Abdullah, M. V. Ramana, C. Y. Khor, K. A. Ahmad, M. A. Mujeebu, Y. Ooi, and M. Z. Ripin, "Numerical and experimental investigations on effect of fan height on the performance of piezoelectric fan in microelectronic cooling," International Communications in Heat and Mass Transfer, 2009, pp. 51-58.

[11] S. M. Wait, S. Basak, S. V. Garimella, A. Raman, "Piezoelectric Fans Using Higher Flexural Modes for Electronics Cooling Applications," IEEE Transactions on Components and Packaging Technologies, 2007, Vol.30, pp. 119-128.

[12] S. F. Sufian, Z. M. Fairuz, M. Zubair, M. Z. Abdullah, and J. J. Mohamed, "Thermal analysis of dual piezoelectric fans for cooling multi-LED packages," Microelectronics Reliability, Vol. 54, Issue 8, pp. 1534-1543, August 2014.

[13] H.K. Ma, L.K. Tan, Y.T. Li, C.L. Liu, "Optimum thermal resistance of the multiple piezoelectric-magnetic fan system," International Communications in Heat and Mass Transfer, Vol. 55, pp. 77-83, July 2014.

[14] Fluent 13 User's Guide, 2012, Fluent Inc. 